\title{
A complex variable solution of two-dimensional heat conduction of composites reinforced with periodic arrays of cylindrically orthotropic fibers
}

\author{
P. Yan ${ }^{a}$, C.P. Jiang ${ }^{a, b, *}$, F. Song ${ }^{b}$ \\ ${ }^{a}$ School of Aeronautic Science and Engineering, Beijing University of Aeronautics and Astronautics, 100191 Beijing, China \\ ${ }^{\mathrm{b}}$ State Key Laboratory of Nonlinear Mechanics (LNM), Institute of Mechanics, Chinese Academy of Sciences, 100190 Beijing, China
}

\section{A R T I C L E I N F O}

\section{Article history:}

Received 22 May 2010

Received in revised form 25 September 2010

Accepted 29 September 2010

Available online 23 October 2010

\section{Keywords:}

Effective thermal conductivity

Cylindrical orthotropy

Complex variable method

Double period

Fiber reinforced composites

\begin{abstract}
A B S T R A C T
Problems of two-dimensional steady-state heat conduction for composites with doubly periodic arrays of cylindrically orthotropic fibers are dealt with. A new complex variable method is presented by introducing an appropriate coordinate transformation to convert the governing differential equation into a harmonic one, and the eigenfunction expansions of the field variables in a unit cell are derived. Then by using a generalized variational functional which absorbs the periodicity condition, an eigenfunction expansion-variational method based on a unit cell is developed to solve such problems. A convergence analysis and a comparison with finite element calculations are conducted to demonstrate the correctness and efficiency of the present method. A discussion is made about the effects of the cylindrical orthotropy of the fiber and the existence of the isotropic core in the fiber on the effective conductivity of the composite. An engineering equivalent parameter, which reflects the overall influence of the thermal conductivities of the matrix and fibers as well as the interfacial characteristic on the effective thermal conductivity of the composite, is found. It is shown that the present first-order approximation of the effective thermal conductivity of the composite can be written in a unified formula for different microstructural characteristics and possesses a good engineering accuracy.
\end{abstract}

(c) 2010 Elsevier B.V. All rights reserved.

\section{Introduction}

Fiber reinforced composites, especially carbon fiber reinforced composites, possess some remarkable properties, thus have been used extensively in many applications from aerospace craft to electric devices. The carbon/epoxy composites have small specific mass, high rupture resistance, very good fatigue strength and good thermal/electrical conductivity. They have been used in the wings, fuselages, horizontal stabilizers, vertical stabilizers, ailerons and so on of airplanes such as Boeing B-787 and Airbus A-380 [1]. The carbon/carbon composites can withstand high-temperature thus be utilized in a variety of high-temperature aerospace applications.

Different from common fibers, carbon fibers possess special heterogeneity and anisotropy [2,3]. Polyacrylonitrile (PAN) based fibers generally exhibit circumferential alignment of the graphite basal planes, while pitch-based fibers typically have radial alignment. Random orientation of the graphite basal planes in the transverse plane of the fiber would result in a transversely isotropic fiber, or in a narrow zone of the fiber would result in a transversely isotropic core in the fiber $[4,5]$. The fibers with such microstructures

\footnotetext{
* Corresponding author at: School of Aeronautic Science and Engineering, Beijing University of Aeronautics and Astronautics, 100191 Beijing, China. Tel.: +86 010 82317508.

E-mail address: jiangchiping@buaa.edu.cn (C.P. Jiang).
}

can be idealized as cylindrically orthotropic fibers with a transversely isotropic core, if any. Several researches about cylindrically orthotropic fibers or cylinders were carried out based on this model. Knott and Herakovich [5] considered the existence of the isotropic core in the effective elastic properties of fiber composites. Rodriguez and Cabeza [6] studied the combination of the effects of the core with the cylindrical orthotropy of the fiber on the effective thermal conductivity using a finite element method. Tarn [7] examined the issue of stress singularity in an elastic cylinder of cylindrically anisotropic materials through a compound cylinder in which the outer cylinder is cylindrically anisotropic and the core is transversely isotropic. Besides carbon fibers, cylindrically anisotropic materials include tree trunks and manufactured composites. Many mathematical methods are developed to analyze performance and behavior of this kind of special materials [8-10] or composites with such kind of fibers [11-13].

Thermal conductivity is a very important property for the applications of fiber reinforced composites, thus lots of researches focused on this topic. Predicting the effective properties from the microstructure of composites is a supplement or a substitution of measurements, and can be used to design composites. Several methods were developed to investigate the thermal/transport properties of composites with cylindrically orthotropic fibers. Benveniste et al. [14] and Hasselman et al. [15] extended the methods of studying the heat conduction of the transversely isotropic 


\begin{tabular}{|c|c|c|c|}
\hline EEVM & eigenfunction expansion-variational method & $X_{n}$ & eigenfunction expansion coefficients \\
\hline & arbitrary arc with two ends $A$ and $B$ & $x_{1}, x_{2}$ & Cartesian coordinates \\
\hline$f, o, m$ & fiber, core and matrix, subscript or superscript & $z$ & $x_{1}+i x_{2}$, complex variable in the $z$-plane \\
\hline & imaginary unit & $z_{1}$ & $r e^{i \theta_{1}}$, complex variable in the $z_{1}$-plane \\
\hline$C_{m}, D_{m n}$ & defined by Eq. (32) & $z_{2}$ & $r^{\gamma-1} z$, complex variable in the $z_{2}$-plane \\
\hline$E_{n}, F_{n}, G_{n}$ & $P_{n}, Q_{n}$ complex coefficients of expansions & $\mathbf{d}_{1}, \mathbf{d}_{2}$ & two fundamental periods of a doubly periodic array \\
\hline$H_{r}, H_{\theta}$ & radial and tangential temperature gradients & & temperature gradient vector \\
\hline$H$ & $\begin{array}{l}\text { thermal contact conductivity across the fiber-matrix } \\
\text { interface }\end{array}$ & $\begin{array}{l}\langle\mathbf{H}\rangle,\left\langle H_{j}\right\rangle \\
\mathbf{n}\end{array}$ & $\begin{array}{l}\text { average temperature gradient vector } \\
\text { unit normal vector on the boundary }\end{array}$ \\
\hline$k_{f}$ & $\begin{array}{l}\sqrt{k_{r} k_{\theta}} \text {, effective thermal conductivity of a cylindrically } \\
\text { orthotropic fiber }\end{array}$ & $\begin{array}{l}\mathbf{p}^{s}, p_{i}^{s} \\
\mathbf{q}\end{array}$ & $\begin{array}{l}\text { translation vector from a boundary } s-\text { to } s^{+} \\
\text {heat flux vector }\end{array}$ \\
\hline$k_{o}, k_{m}$ & conductivities of core and matrix & $\mathbf{x}$ & position vector \\
\hline$k_{r}, k_{\theta}$ & radial and tangential conductivities & $\alpha$ & nondimensional parameter, defined by Eq. (35) \\
\hline$k_{e}$ & $\begin{array}{l}\text { effective thermal conductivity of a transversely isotro- } \\
\text { pic composite }\end{array}$ & $\begin{array}{l}\beta \\
\delta(\cdot)\end{array}$ & $\begin{array}{l}\left(k_{m} /(2 h R), \text { nondimensional parameter }\right. \\
\text { variation }\end{array}$ \\
\hline$k_{i j}^{e}$ & effective thermal conductivity tensor of a composite & $\Phi$ & heat transfer rate through an arbitrary arc \\
\hline$L^{j}$ & side length of a unit cell & $\gamma$ & $\sqrt{k_{\theta} / k_{r}}$ \\
\hline$N$ & term number of eigenfunction expansion & $\eta_{f m}$ & $\left(k_{m}-k_{f}\right) /\left(k_{m}+k_{f}\right)$, nondimensional parameter \\
\hline$m, n$ & serial number & $\eta_{o f}$ & $\left(k_{f}-k_{o}\right) /\left(k_{f}+k_{o}\right)$, nondimensional parameter \\
\hline$q$ & q $\cdot \mathbf{n}$, boundary heat flux & $\eta_{n}$ & defined by Eqs. (26) and (43) \\
\hline $\begin{array}{l}q_{r}, q_{\theta} \\
q_{1}, q_{2}\end{array}$ & $\begin{array}{l}\text { radial and tangential components of heat flux field } \\
x_{1} \text { and } x_{2} \text { components of heat flux field }\end{array}$ & $\eta$ & $\begin{array}{l}\text { nondimensional parameter, defined by Eqs. (35) } \\
\text { and (36) }\end{array}$ \\
\hline $\begin{array}{l}\left\langle q_{i}\right\rangle \\
R\end{array}$ & average heat flux vector within the unit cell & $\lambda$ & volume fraction of the fiber including its core \\
\hline $\begin{array}{l}\kappa_{0}, K \\
r, \theta\end{array}$ & $\begin{array}{l}\text { radii of the core and fiber cross-sections } \\
\text { polar coordinates }\end{array}$ & $\Pi$ & functional, defined by Eq. (15) \\
\hline $\begin{array}{l}1, \theta \\
s\end{array}$ & serial number of a unit cell boundary & & $\theta / \gamma$, polar coordinate in the $z_{1}$-plane \\
\hline T & $\begin{array}{l}\text { serial number or a unit cell Doundary } \\
\text { temperature }\end{array}$ & $\omega\left(z_{2}\right)$ & generalized complex potential in the $z_{2}$-plane \\
\hline$\partial V^{+}$ & positive boundary of a unit cell & $\omega(z)$ & tial in the $z$-plane \\
\hline$\partial V^{-}$ & negative boundary of a unit cell & $\xi_{0}$ & lative radius of core cross-section \\
\hline
\end{tabular}

fiber composites to cylindrically orthotropic fiber composites. These methods are approximate in treating inclusion interactions. On the other hand, a model of periodic array of fibers is used to consider the inclusion interactions. Rodriguez and Cabeza [6] presented a unit cell model of finite elements to study the effective thermal conductivity of fiber composites. Chen and Kuo [16] generalized Rayleigh's method to account for the periodic arrangement of cylindrically orthotropic and exponentially graded cylinders.

For a steady-state heat conduction problem of composite with a doubly periodic array of fibers, the temperature field is quasi-periodic and the heat flux field is periodic, which is similar to the elasticity issue [17]. Thus, a corresponding periodic boundary condition should be prescribed in the analysis based on a unit cell model. Xia et al. [17] and Jiang et al. [18] pointed out that the effective moduli/conductivities obtained under periodic boundary conditions are bounded by those obtained under homogeneous displacement/temperature boundary conditions and homogeneous traction/flux boundary conditions, hence are more reasonable.

This paper is devoted to presenting a new complex variable method for two-dimensional steady-state heat conduction problems of cylindrically orthotropic fibers. Then the new complex variable method is used to develop an eigenfunction expansionvariational method based on a unit cell for analysis of such composites, in terms of which some interesting and concise laws hidden in the complicated relation between the macroscopic thermal properties and microstructures are revealed. A useful engineering equivalent parameter is found, and a convenient and efficient engineering closed-form formula is provided.

The paper is organized as follows. Section 2 presents a new complex variable method for the problem under consideration, then the field variables are formulated by a generalized complex potential. Section 3 constructs a generalized variational functional which absorbs the doubly periodic boundary conditions of a unit cell. Section 4 gives the expansions of the complex potentials in the matrix, cylindrically orthotropic fiber and isotropic core, respectively. The interfacial conditions are used to derive the relations between the unknown coefficients. Then an eigenfunction expansion of the complex potential is derived. Section 5 determines the remaining unknown coefficients by using the generalized variational functional. Section 6 determines the effective thermal conductivity of such a composite and a unified first-order approximation formula is obtained. An engineering equivalent parameter which reflects the overall influence of the thermal conductivities of the matrix and fibers as well as the interfacial characteristic on the effective thermal conductivity of the composite, is found. In Sections 7.1 and 7.2, a qualitative analysis and a quantitative analysis of the effects of the cylindrical orthotropy of fibers as well as the existence of a core on the microscopic fields and macroscopic thermal properties are presented. The numerical results show that the unified first-order approximation formula possesses a good engineering accuracy. Lastly, several concluding remarks are made in Section 8.

\section{Generalized complex potential}

Consider a cylindrically orthotropic fiber under twodimensional steady-state heat conduction as shown in Fig. 1, where $\left(x_{1}, x_{2}\right)$ are the Cartesian coordinates and $(r, \theta)$ are the polar coordinates. The temperature $T$, heat fluxes $\left\{q_{r}, q_{\theta}\right\}$ and temperature gradients $\left\{H_{r}, H_{\theta}\right\}$ in the polar coordinates satisfy following equations:

$\left[\begin{array}{l}q_{r} \\ q_{\theta}\end{array}\right]=-\left[\begin{array}{cc}k_{r} & 0 \\ 0 & k_{\theta}\end{array}\right]\left[\begin{array}{l}H_{r} \\ H_{\theta}\end{array}\right]$ 


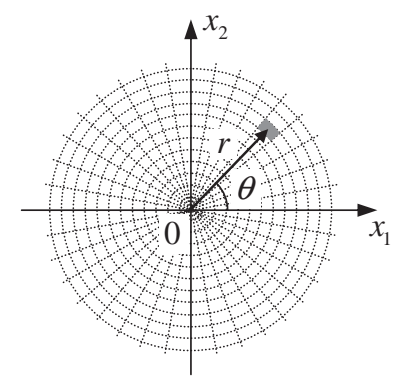

Fig. 1. Cross-section of a cylindrically orthotropic fiber.

$\left[\begin{array}{l}H_{r} \\ H_{\theta}\end{array}\right]=\left[\begin{array}{c}\frac{\partial T}{\partial r} \\ \frac{1}{r} \frac{\partial T}{\partial \theta}\end{array}\right]$

$\frac{\partial q_{r}}{\partial r}+\frac{q_{r}}{r}+\frac{\partial q_{\theta}}{r \partial \theta}=0$

where $k_{r}$ and $k_{\theta}$ are the radial and tangential conductivities, respectively. If $k_{r}>k_{\theta}$, the material is called radially orthotropic, and if $k_{r}<k_{\theta}$, it is circumferentially orthotropic [14].

The substitution of Eqs. (1) and (2) into Eq. (3) yields the following governing differential equation:

$\frac{\partial}{\partial r}\left(r \frac{\partial T}{\partial r}\right)+\gamma^{2} \frac{1}{r} \frac{\partial^{2} T}{\partial \theta^{2}}=0$

where $\gamma=\sqrt{k_{\theta} / k_{r}}$. Let the origin of a complex plane:

$z=x_{1}+i x_{2}=r e^{i \theta}$

be located at the center of the cross-section of a fiber as shown in Fig. 2a. In a general case of $\gamma \neq 1$ or $k_{\theta} \neq k_{r}$, Eq. (4) is not a harmonic equation. In order to develop a complex variable method, introduce a transformation

$z_{1}=r e^{i \theta_{1}}$

where $\theta_{1}=\theta / \gamma$. In the $z_{1}$-plane, the intact circular region of the fiber is mapped onto a circular region with a fan notch as shown in Fig. 2b, and the governing Eq. (4) is transformed into a harmonic equation:

$\frac{\partial}{\partial r}\left(r \frac{\partial T}{\partial r}\right)+\frac{1}{r} \frac{\partial^{2} T}{\partial \theta_{1}^{2}}=0$

It is worth noting that the transformation from the $z$-plane to the $z_{1}$-plane is not a conformal mapping. Introduce again a conformal mapping from the $z_{1}$-plane to the $z_{2}$-plane:

$z_{2}=z_{1}^{\gamma}=r^{\gamma-1} z$
The circular region with a fan notch in Fig. 2b is conformally mapped onto an intact circular region in the $z_{2}$-plane as shown in Fig. 2c. In the $z_{2}$-plane, the temperature field is still a harmonic function and can be formulated by a generalized complex potential $\omega\left(z_{2}\right)$ :

$T=\frac{1}{2}\left[\omega\left(z_{2}\right)+\overline{\omega\left(z_{2}\right)}\right]$

where the upper bar denotes the conjugate. The substitution of Eq. (9) into Eqs. (1) and (2) yields:

$$
\left[\begin{array}{l}
q_{r} \\
q_{\theta}
\end{array}\right]=-\left[\begin{array}{c}
k_{r} \frac{\gamma}{r} \cdot \frac{1}{2}\left[\omega^{\prime}\left(z_{2}\right) z_{2}+\overline{\omega^{\prime}\left(z_{2}\right) z_{2}}\right] \\
-k_{\theta} \frac{1}{r} \cdot \frac{1}{2 i}\left[\omega^{\prime}\left(z_{2}\right) z_{2}-\overline{\omega^{\prime}\left(z_{2}\right) z_{2}}\right]
\end{array}\right]
$$

The heat transfer rate $\Phi$ through an arbitrary arc, AB, can be calculated by integrating the heat flux

$\Phi=-\sqrt{k_{r} k_{\theta}} \frac{1}{2 i}\left[\omega\left(z_{2}\right)-\overline{\omega\left(z_{2}\right)}\right]_{\mathrm{A}}^{\mathrm{B}}$

Thus all the field variables in a cylindrically orthotropic fiber are formulated by a generalized complex potential $\omega\left(z_{2}\right)$. In a (transversely) isotropic material, $\gamma=1$, Eqs. (9)-(11) degenerate into:

$T=\frac{1}{2}[\omega(z)+\overline{\omega(z)}]$

$q_{r}-i q_{\theta}=-k \frac{1}{r} \omega^{\prime}(z) z$ in the polar coordinates

$q_{1}-i q_{2}=-k \omega^{\prime}(z)$ in the rectangular coordinates

$\Phi=\frac{-k}{2 i}[\omega(z)-\overline{\omega(z)}]_{\mathrm{A}}^{\mathrm{B}}$

where the complex potential $\omega(z)$ is well known.

\section{Generalized variational functional for a unit cell}

Consider a composite with a doubly periodic array of cylindrically orthotropic fibers. Two fiber arrays of practical importance, i.e. the hexagonal array and square array, are shown in Fig. 3a and $b$, respectively, where $\mathbf{d}_{1}$ and $\mathbf{d}_{2}$ denote two fundamental periods. According to periodicity, the cell boundary can be divided into $\partial V^{+}=\sum_{s} \partial V_{s}^{+}$and $\partial V^{-}=\sum_{s} \partial V_{s}^{-}$, where $s=1,2,3$ in Fig. 3a and $s=1,2$ in Fig. $3 \mathrm{~b}$. For steady-state heat conduction, the temperature field $T(\mathbf{x})$ is quasi-periodic, and the heat flux field $\mathbf{q}(\mathbf{x})$ and the temperature gradient field $\mathbf{H}(\mathbf{x})$ are periodic. The thermal loading condition of a unit cell can be prescribed by an average temperature gradient $\langle\mathbf{H}\rangle$. By using the Lagrangian multiplier method, the periodic boundary condition of a unit cell can be incorporated into the functional for heat conduction:

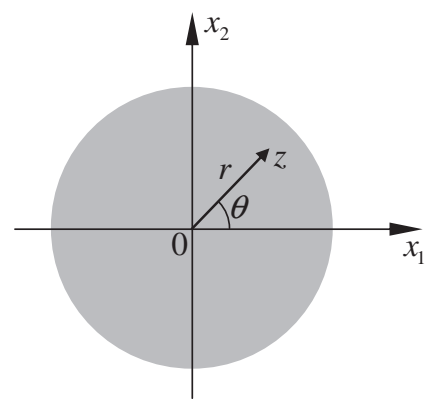

(a) $z$ plane

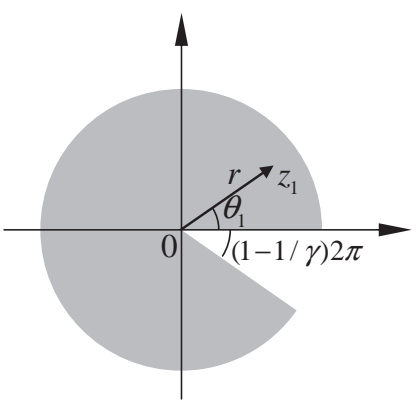

(b) $z_{1}$ plane

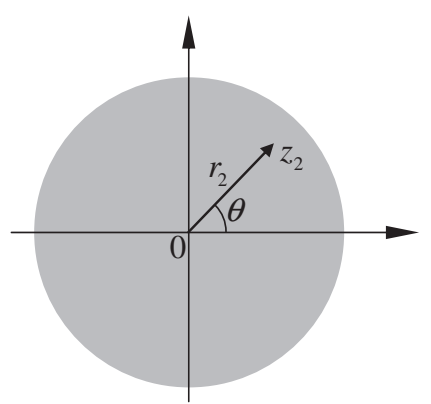

(c) $z_{2}$ plane

Fig. 2. Transformation of coordinates. (a) $z$-plane, (b) $z_{1}$-plane and (c) $z_{2}$-plane. 
(a)

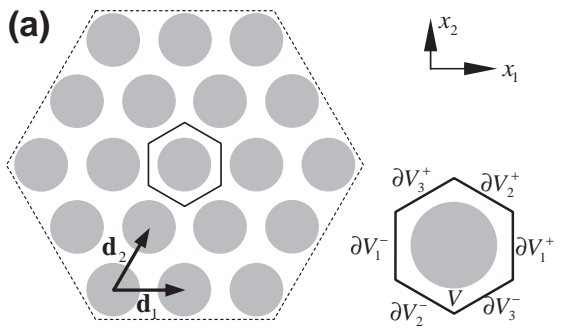

(b)

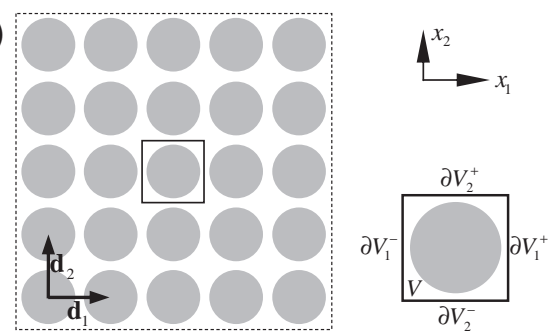

Fig. 3. Hexagonal and square arrays of fibers and their symmetrical unit cells.

$\Pi=\int_{V} \frac{1}{2} \mathbf{q} \cdot \mathbf{H} d V-\sum_{s} \int_{\partial V_{s}^{+}} q^{s+} \cdot\left(T^{s+}-T^{S-}-\langle\mathbf{H}\rangle \cdot \mathbf{p}^{s}\right) d S$

where $q(=\mathbf{q} \cdot \mathbf{n})$ denotes the boundary heat flux, $\mathbf{n}$ denotes the unit normal vector on the boundary; the quantities with superscripts " $s-$ " and "s+" are corresponding to taking values from $\partial V_{s}^{-}$and $\partial V_{s}^{+}$, respectively; $\mathbf{p}^{1}=\mathbf{d}_{1}, \mathbf{p}^{2}=\mathbf{d}_{2}$ and $\mathbf{p}^{3}=\mathbf{d}_{2}-\mathbf{d}_{1}$ in Fig. 3a and $\mathbf{p}^{1}=\mathbf{d}_{1}$ and $\mathbf{p}^{2}=\mathbf{d}_{2}$ in Fig. $3 \mathrm{~b}$.

The stationary condition [19] of the functional (15) can be written as:

$$
\begin{aligned}
& \sum_{S} \int_{\partial V_{s}^{+}} \delta q^{s+}\left(T^{s+}-T^{s-}\right) d S-\sum_{S} \int_{\partial V_{s}^{+}}\left(q^{s+}+q^{s-}\right) \delta T^{s-} d S \\
& =\sum_{S} \int_{\partial V_{s}^{+}} \delta q^{s+}\langle\mathbf{H}\rangle \cdot \mathbf{p}^{s} d S
\end{aligned}
$$

where $\delta(\cdot)$ denotes the variation.

The stationary condition (16) of the generalized variational functional can be used to develop an eigenfunction expansion-variational method based on a unit cell to solve the problem of steadystate heat conduction.

\section{Eigenfunction expansions in a unit cell}

A typical unit cell of a composite with a doubly periodic array of cylindrically orthotropic fibers is divided into three regions occupied, respectively, by a cylindrically orthotropic fiber, a transversely isotropic fiber core and a surrounding isotropic matrix as shown in Fig. 4. This section deals with the expansions of the complex potentials, $\omega_{f}\left(z_{2}\right), \omega_{o}(z)$ and $\omega_{m}(z)$, in the three regions, where the subscripts " $f$ ", " $o$ " and " $m$ " refer to the fiber, core and matrix, respectively. It is seen that the expansion of $\omega_{f}\left(z_{2}\right)$ in the $z_{2}$-plane can be transformed to the $z$-plane by using Eq. (8).

In the matrix region, the complex potential $\omega_{m}(z)$ is expanded into a Laurent series: $\omega_{m}(z)=\sum_{n=1}^{\infty} G_{n} z^{-(2 n-1)}+\sum_{n=1}^{\infty} F_{n} z^{2 n-1}$

where $G_{n}$ and $F_{n}$ are complex coefficients, where only odd terms remain due to the centrosymmetry of the unit cell.

The complex potential $\omega_{o}(z)$ in the transversely isotropic core can be expanded into a Taylor series:

$\omega_{o}(z)=\sum_{n=1}^{\infty} E_{n} z^{2 n-1}$

where $E_{n}$ is a complex coefficient. The complex potential $\omega_{f}\left(z_{2}\right)$ can be expanded into a Laurent series in the $z_{2}$-plane, then be transformed to the $z$-plane

$$
\begin{aligned}
\omega_{f}\left(z_{2}\right) & =\sum_{n=1}^{\infty} P_{n} z_{2}^{-(2 n-1)}+\sum_{n=1}^{\infty} Q_{n} z_{2}^{2 n-1} \\
& =\sum_{n=1}^{\infty} P_{n} r^{(\gamma-1)(1-2 n)} z^{1-2 n}+\sum_{n=1}^{\infty} Q_{n} r^{(\gamma-1)(2 n-1)} z^{2 n-1}
\end{aligned}
$$

where $P_{n}$ and $Q_{n}$ are complex coefficients

Now examine the relation between five sets of unknown coefficients, $G_{n}, F_{n}, E_{n}, P_{n}$ and $Q_{n}$.

Assuming the fiber-core interface is perfect and the fiber-matrix interface is imperfect, the continuity and jump discontinuity conditions across the two interfaces can be written as:

$T_{o}=T_{f}, \Phi_{o}=\Phi_{f} \quad$ at $|z|=R_{0}$

$q_{f}=q_{m}=-h\left(T_{f}-T_{m}\right), \Phi_{f}=\Phi_{m} \quad$ at $|z|=R$

where $h$ is the thermal contact conductivity across the fiber-matrix interface, $R_{0}$ and $R$ denote the radii of the core and fiber crosssections, respectively. Substituting Eqs. (17)-(19) into Eqs. (9)(11) and Eqs. (12)-(14), and then into Eqs. (20) and (21), one obtains:
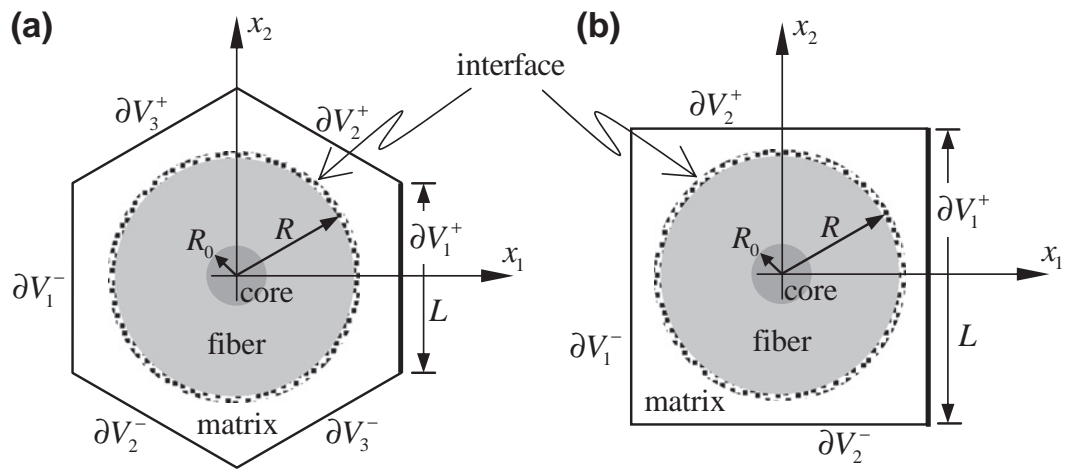

Fig. 4. Two kinds of unit cells containing a cylindrically orthotropic fiber with an isotropic core. (a) Hexagonal unit cell and (b) square unit cell. 
$E_{n}=\left(1+\eta_{o f}\right) R_{0}^{(\gamma-1)(2 n-1)} Q_{n}$

$P_{n}=\eta_{o f} R_{0}^{2 \gamma(2 n-1)} \bar{Q}_{n}$

$F_{n}-\bar{G}_{n} R^{2-4 n}=\frac{1-\eta_{f m}}{1+\eta_{f m}}\left(Q_{n} R^{(\gamma-1)(2 n-1)}-\bar{P}_{n} R^{(\gamma+1)(1-2 n)}\right)$

$G_{n}=\eta_{n} R^{4 n-2} \bar{F}_{n}$

where

$\eta_{n}=\frac{\eta_{f m}+\eta_{o f} \xi_{0}^{2 \gamma(2 n-1)}+(2 n-1) \beta\left(1-\eta_{f m}\right)\left(1-\eta_{o f} \xi_{0}^{2 \gamma(2 n-1)}\right)}{1+\eta_{f m} \eta_{o f} \xi_{0}^{2 \gamma(2 n-1)}+(2 n-1) \beta\left(1-\eta_{f m}\right)\left(1-\eta_{o f} \xi_{0}^{2 \gamma(2 n-1)}\right)}$

and $\quad \eta_{f m}=\left(k_{m}-k_{f}\right) /\left(k_{m}+k_{f}\right), \quad \eta_{o f}=\left(k_{f}-k_{o}\right) /\left(k_{f}+k_{o}\right), \quad \beta=k_{m} /(2 h R)$, $\xi_{0}=R_{0} / R, k_{f}\left(=\sqrt{k_{r} k_{\theta}}\right)$ is called the effective thermal conductivity of a cylindrically orthotropic fiber. Thus the eigenfunction expansion of the complex potential $\omega_{m}(z)$ can be written as:

$\omega_{m}(z)=\sum_{n=1}^{\infty} \eta_{n} R^{4 n-2} \bar{F}_{n} z^{-(2 n-1)}+\sum_{n=1}^{\infty} F_{n} z^{2 n-1}$

Consider the square and hexagonal unit cells shown in Fig. 4. If the boundary condition is also symmetric about $x_{2}$-axis, the expansion coefficients additionally satisfy following relations:

$\bar{E}_{n}=-E_{n}, \bar{P}_{n}=-P_{n}, \bar{Q}_{n}=-Q_{n}, \bar{G}_{n}=-G_{n}, \bar{F}_{n}=-F_{n}$

i.e. the expansion coefficients are pure imaginary. The eigenfunction expansion of the complex potential $\omega_{m}(z)$ is further simplified as:

$\omega_{m}(z)=\sum_{n=1}^{\infty} F_{n}\left[-\eta_{n} R^{4 n-2} z^{-(2 n-1)}+z^{2 n-1}\right]$

The remaining work is to determine a set of unknown coefficients, $F_{n}$, which can be completed by using the stationary condition (Eq. (16)).

\section{Determination of the unknown coefficients in the eigenfunction expansion}

In this section, a detailed solving procedure of the unknown coefficients is given for the case of the heat flux being symmetric about $x_{2}$-axis. Substituting Eq. (29) into Eqs. (12)-(14), and taking an appropriate truncation of each expansion, the heat flux, temperature and heat transfer rate can be expressed as follows:

$q_{i}=\sum_{n=1}^{N} X_{n} q_{i}^{(n)}, T=\sum_{n=1}^{N} X_{n} T^{(n)}, \Phi=\sum_{n=1}^{N} X_{n} \Phi^{(n)} \quad i=1,2$

where
$\sum_{n=1}^{N} D_{m n} X_{n}=C_{m} \quad m=1,2, \ldots, N$

where

$$
\begin{aligned}
D_{m n}= & \sum_{s} \int_{\partial V_{s}^{+}} \mathbf{n}^{s+} \cdot \mathbf{q}_{(m)}^{s+}\left(T_{(n)}^{s+}-T_{(n)}^{s-}\right) d S \\
& -\sum_{s} \int_{\partial V_{s}^{+}} \mathbf{n}^{s-} \cdot\left(\mathbf{q}_{(n)}^{s-}-\mathbf{q}_{(n)}^{s+}\right) T_{(m)}^{s-} d S
\end{aligned}
$$

$C_{m}=\sum_{s} \int_{\partial V_{s}^{+}}\left(\mathbf{n}^{s+} \cdot \mathbf{q}_{(m)}^{s+}\right)\left(\langle\mathbf{H}\rangle \cdot \mathbf{p}^{s}\right) d S=\sum_{s}\left[\Phi_{(m)}^{s+}\langle\mathbf{H}\rangle \cdot \mathbf{p}^{s}\right]$

$\mathbf{q}_{(m)}^{s+}, T_{(n)}^{s+}$ and $\Phi_{(m)}^{s+}$ denote taking the values of $\mathbf{q}^{(m)}, T^{(n)}$ and $\Phi^{(m)}$ from $\partial V_{s}^{+}$, respectively, and the quantities with the superscript " $s-$ " are corresponding to taking values from $\partial V_{s}^{-}$.

Once the unknown coefficients are determined by Eq. (32), the heat flux and temperature fields can be obtained by Eq. (30).

\section{Effective thermal conductivities}

The effective thermal conductivities of a composite, $k_{i j}^{e}$, are determined with the aid of the average field theory

$\left\langle q_{i}\right\rangle=-k_{i j}^{e}\left\langle H_{j}\right\rangle$

where $\left\langle q_{i}\right\rangle$ is the average heat flux within the unit cell, which can be calculated by the following formula:

$\left\langle q_{i}\right\rangle=\frac{1}{V} \int_{V} q_{i} d V=\frac{1}{V} \sum_{s} \Phi^{s+} p_{i}^{s}$

where $\Phi^{S+}$ is the heat transfer rate through the boundary $\partial V_{s}^{+}$. It is worth noting that the periodic boundary conditions are prescribed for the unit cell by setting the average temperature gradient $\langle\mathbf{H}\rangle$, and then the average heat flux are solved for calculating the effective thermal conductivities. When the term number $N$ of the eigenfunction expansion is large enough, high-order numerical results of the effective thermal conductivities are obtained; when $N=1$, a first-order approximation formula is obtained.

For a composite with a square or hexagonal array of fibers, the effective conductivity is transversely isotropic, that is $k_{11}^{e}=k_{22}^{e}=$ $k_{e}$. Interestingly, for these two fiber arrays, the first-order approximation formula of the nondimensional effective conductivity can be written as a unified expression:

$\frac{k_{e}}{k_{m}}=\frac{(\pi-\alpha \eta \lambda)^{2}}{2 \pi^{2}(1+\eta \lambda)-(\pi+\alpha \eta \lambda)^{2}}$

where $\lambda$ is the volume fraction of the fiber including its core; and
$1 \leqslant n \leqslant N$

$1 \leqslant n \leqslant N$

$1 \leqslant n \leqslant N$

$1 \leqslant n \leqslant N$

$1 \leqslant n \leqslant N$
The substitution of Eq. (30) into the stationary condition (16) yields the following linear algebraic equations: $\eta=\frac{\eta_{f m}+\eta_{o f} \xi_{0}^{2 \gamma}+\beta\left(1-\eta_{f m}\right)\left(1-\eta_{o f} \xi_{0}^{2 \gamma}\right)}{1+\eta_{f m} \eta_{o f} \xi_{0}^{2 \gamma}+\beta\left(1-\eta_{f m}\right)\left(1-\eta_{o f} \xi_{0}^{2 \gamma}\right)}$ 
$\alpha= \begin{cases}2 & \text { for square array } \\ 3 \sqrt{3} / 2 & \text { for hexagonal array }\end{cases}$

Eq. (35b) can degenerate into following three special cases:

$\eta=\left\{\begin{array}{cc}\frac{\eta_{f m}+\eta_{\text {of }} \xi_{0}^{2 \gamma}}{1+\eta_{f m} \eta_{o f} \xi_{0}^{2 \gamma}} & \text { with core, perfect fiber-matrix intrerface } \\ \frac{\eta_{f m}+\beta\left(1-\eta_{f m}\right)}{1+\beta\left(1-\eta_{f m}\right)} & \text { without core, imperfect fiber-matrix intrerface } \\ \eta_{f m} & \text { without core, perfect fiber-matrix intrerface }\end{array}\right.$

From Eqs. (35) and (36), it is seen that the present analytical method reveals an engineering equivalent parameter of practical importance, $\eta$, which can reflect the overall influence of the thermal conductivities of the matrix and fibers as well as the interfa- where

$$
\begin{aligned}
& E_{n}=\left(1+\eta_{o f}\right) R_{0}^{(\gamma-1)(2 n-1)} Q_{n}, \quad P_{n}=-\eta_{o f} R_{0}^{2 \gamma(2 n-1)} Q_{n}, \\
& i Q_{n}=\frac{1+\eta_{f m}}{1-\eta_{f m}} \cdot \frac{\left(1-\eta_{n}\right) R^{(\gamma-1)(1-2 n)}}{1-\eta_{o f} \xi_{0}^{2 \gamma(2 n-1)}} X_{n}
\end{aligned}
$$

First examine the effects of the existence of the core and the cylindrical orthotropy of the fiber on the heat flux at the center of the fiber. If a transversely isotropic core exists in a central region of the fiber, it is seen from Eq. (37) that the heat flux at $r=0$ is finite:

$$
\left[\begin{array}{l}
q_{r}^{o} \\
q_{\theta}^{o}
\end{array}\right]_{r=0}=-k_{o}\left(i E_{1}\right)\left[\begin{array}{c}
\sin \theta \\
\cos \theta
\end{array}\right]
$$

If no transversely isotropic core exists in the fiber, i.e. $R_{0}=0$, Eq. (38) is reduced to:

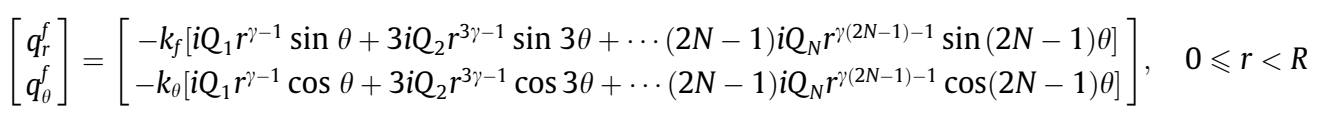

cial characteristic on the effective thermal conductivity of the composites with a good engineering accuracy. The equivalent parameter greatly simplifies the complicated relation of the effective thermal conductivity to the internal structure of a composite. By using the equivalent parameter $\eta$, the present solutions provide a unified first-order approximation formula of the effective thermal conductivity for different microstructural characteristics. The numerical analysis in the next section will show that the first-order approximation formula can serve as a convenient and efficient engineering closed-form formula with a good engineering accuracy.

\section{Analysis and discussion}

To understand profoundly the effect of the cylindrical orthotropy of the fiber and the existence of the core, as well as to demonstrate applications of the present method, a qualitative analysis and a quantitative analysis are presented in Sections 7.1 and 7.2, respectively.

\subsection{Qualitative analysis}

From Eqs. (10), (13), (17)-(19) the heat flux fields in the core, fiber and matrix can be expressed as:

$$
\left[\begin{array}{l}
q_{r}^{o} \\
q_{\theta}^{o}
\end{array}\right]=-k_{o} \sum_{n=1}^{N}\left(i E_{n}\right)(2 n-1) r^{2 n-2}\left[\begin{array}{l}
\sin (2 n-1) \theta \\
\cos (2 n-1) \theta
\end{array}\right], \quad 0 \leqslant r<R_{0}
$$

$$
\left[\begin{array}{c}
q_{r}^{f} \\
q_{\theta}^{f}
\end{array}\right]=\left[\begin{array}{c}
-k_{f} \sum_{n=1}^{N}\left(i P_{n} r^{\gamma(1-2 n)-1}+i Q_{n} r^{\gamma(2 n-1)-1}\right)(2 n-1) \sin (2 n-1) \theta \\
-k_{\theta} \sum_{n=1}^{N}\left(-i P_{n} r^{\gamma(1-2 n)-1}+i Q_{n} r^{\gamma(2 n-1)-1}\right)(2 n-1) \cos (2 n-1) \theta
\end{array}\right],
$$$$
R_{0} \leqslant r<R
$$

$$
\begin{aligned}
{\left[\begin{array}{l}
q_{1}^{m} \\
q_{2}^{m}
\end{array}\right] } & =\left[\begin{array}{l}
-k_{m} \sum_{n=1}^{N} X_{n}\left(-\eta_{n} R^{4 n-2} r^{-2 n}+r^{2 n-2}\right)(2 n-1) \sin (2 n-1) \theta \\
-k_{m} \sum_{n=1}^{N} X_{n}\left(\eta_{n} R^{4 n-2} r^{-2 n}+r^{2 n-2}\right)(2 n-1) \cos (2 n-1) \theta
\end{array}\right], \\
r & \geqslant R
\end{aligned}
$$

Eq. (42) shows that the heat flux at $r=0$ is related to the value of $\gamma\left(=\sqrt{k_{\theta} / k_{r}}\right)$, which is divided into three cases:

when $\gamma<1$, the radial and circumferential heat fluxes at $r=0$ are singular;

when $\gamma=1$, the radial and circumferential heat fluxes at $r=0$ are finite, $\left\{q_{r}^{f}, q_{\theta}^{f}\right\}_{r=0}=-k_{f}\left(i Q_{1}\right)\{\sin \theta, \cos \theta\}$;

when $\gamma>1$, the radial and circumferential heat fluxes at $r=0$ are equal to zero.

It is worth noting that the above conclusions are independent of fiber distribution.

Then examine the effects of the existence of the core and the cylindrical orthotropy of the fiber on the heat flux in the matrix. From Eqs. (13), (26), and (27), it is seen that the effect of the properties of the fiber and core on the heat flux in the matrix is only related to a single parameter $\eta_{n}$.

If no transversely isotropic core exists in the fiber, that is $\xi_{0}=0$, Eq. (26) can be rewritten as:

$\eta_{n}=\frac{\eta_{f m}+(2 n-1) \beta\left(1-\eta_{f m}\right)}{1+(2 n-1) \beta\left(1-\eta_{f m}\right)} \quad$ without core

In this case, the heat flux in the matrix is related to the geometric mean, $\sqrt{k_{\theta} k_{r}}$, of the radial and tangential conductivities of the fiber. Eqs. (33) and (34) show that the effective conductivity of a composite is only related to the boundary heat flux of a unit cell, or the heat flux in the matrix, so the effective conductivity of a composite is related to $k_{f}\left(=\sqrt{k_{\theta} k_{r}}\right)$. This fact implies that a cylindrically orthotropic fiber can be equivalent to a transversely isotropic fiber with a transverse conductivity $\sqrt{k_{\theta} k_{r}}$. The results given by the first-order approximation formula (Eq. (35)) also lead to this conclusion, which was drawn by Hasselman et al. [15] in their early researches. The present results demonstrate that this conclusion holds for composites with doubly periodic arrays of fibers.

If a transversely isotropic core exists in the central region of the fiber, from Eq. (26) $\eta_{n}$ is related to two parameters $\gamma\left(=\sqrt{k_{\theta} / k_{r}}\right)$ and $k_{f}\left(=\sqrt{k_{\theta} k_{r}}\right)$, so such a cylindrically orthotropic fiber cannot be generally equivalent to a transversely isotropic fiber. However, in two special cases, such an equivalence relation holds. One is that the conductivity of the core satisfies $k_{o}=k_{f}=\sqrt{k_{\theta} k_{r}}$, or $\eta_{\text {of }}=0$, Eq. (26) degenerates into Eq. (43). Another is that $\gamma \gg 1$. Since $\xi_{0}<1, \xi_{0}^{2 \gamma} \approx 0$, Eq. (26) also degenerates into Eq. (43). In the latter case, $E_{n} \approx 0$ in Eq. (37), hence the heat flux in the core approaches zero, which implies that the influence of the core is shielded. 


\subsection{Quantitative analysis}

Take the carbon fiber reinforced aluminoborosilicate glass as the computational object. Such a computation requires the data of the radial and circumferential conductivities of the fiber, however, they are not available and hardly measured by an experiment. To bypass the difficulty, Hasselman et al. [15] presented a reasonable assumption. The carbon fibers are made up of the graphite basal planes on different patterns. One type of the graphitic carbon fiber is made up of concentric cylinders of the graphite basal planes, which is called a circumferentially orthotropic fiber. It is expected that the value of the radial conductivity will correspond to the value of the transverse thermal conductivity $(2.4 \mathrm{~W} / \mathrm{m} \mathrm{K})$ of the single crystal graphite, and the tangential conductivity will correspond to the value of the in-plane thermal conductivity $(100 \mathrm{~W} / \mathrm{m} \mathrm{K})$. Similarly the transverse conductivity of the radially orthotropic fiber can be estimated. The conductivity of the aluminoborosilicate glass matrix is $1.0 \mathrm{~W} / \mathrm{m} \mathrm{K}$. So that:

$k_{r} / k_{m}=2.4, \quad k_{\theta} / k_{m}=100$, for a circumferentially orthotropic fiber;

$k_{r} / k_{m}=100, k_{\theta} / k_{m}=2.4$, for a radially orthotropic fiber.

For the convenience of comparison, meanwhile, the conductivity of a transversely isotropic fiber is taken the effective transverse conductivity of the cylindrically orthotropic fiber:

$$
k_{r} / k_{m}=k_{\theta} / k_{m}=\sqrt{240} \text {, for a transversely isotropic fiber. }
$$

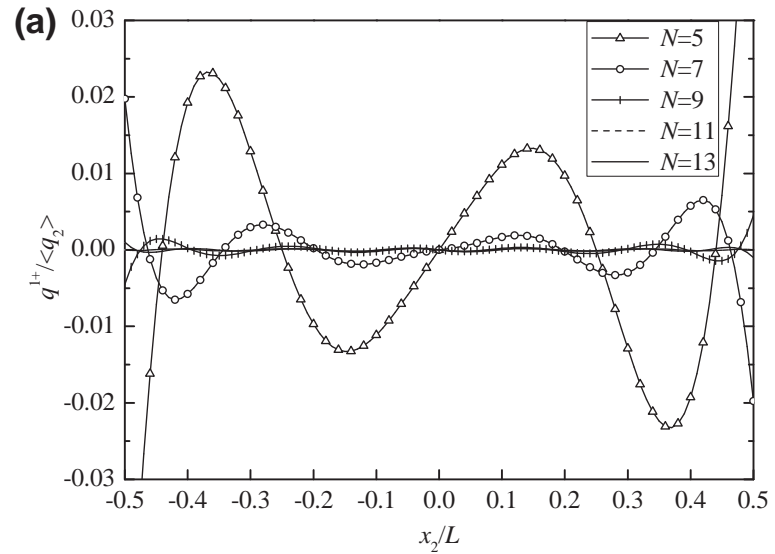

First verify the validity and convergence of the present method.

A periodic boundary condition is prescribed on the unit cell by setting the average temperature gradients: $\left\langle H_{1}\right\rangle=0,\left\langle H_{2}\right\rangle=1$. The boundary heat flux at the boundary $\partial V_{1}^{+}$should be zero $\left(q^{1+}=0\right)$ for the hexagonal and square unit cells shown in Fig. 4 due to the symmetry, which is used to verify the validity and convergence of the present method. The parameters are taken as $k_{r}\left|k_{m}=100, k_{\theta}\right|$ $k_{m}=2.4, k_{\mathrm{o}} / k_{m}=1, \beta=k_{m} /(2 h R)=0.0572, \xi_{0}=R_{0} / R=0.1$, and the volume fraction $\lambda=0.78$, the side length $L=R \sqrt{\pi / \lambda}$ for the square unit cell, and $\lambda=0.9, L=R \sqrt{2 \pi / 3 \sqrt{3}} \lambda$ for the hexagonal unit cell. It is seen from Fig. 5 that the nondimensional boundary heat flux $q^{1+} /\left\langle q_{2}\right\rangle$ approaches zero rapidly with the increase of the eigenfunction expansion term number $N$ even for a very high fiber volume fraction. The numerical results verify the validity and convergence of the present method.

To further verify the convergence and accuracy of the present method, a comparison with Rodriguez and Cabeza's [6] finite element results is listed in Table 1 for a square array of fibers. Rodriguez and Cabeza did not report finite element results for a hexagonal array, which are supplemented by using the commercial software ANSYS and are listed in Table 2. The detailed implementation procedure refers to the one for calculating the effective mechanical properties [17] and the thermoelectroelastic properties [20]. From Tables 1 and 2, a good agreement and rapid convergence of the present method are observed. It is interesting to notice that the present first-order approximation formula $(N=1)$ possesses a good engineering accuracy and can serve as a convenient and effi-

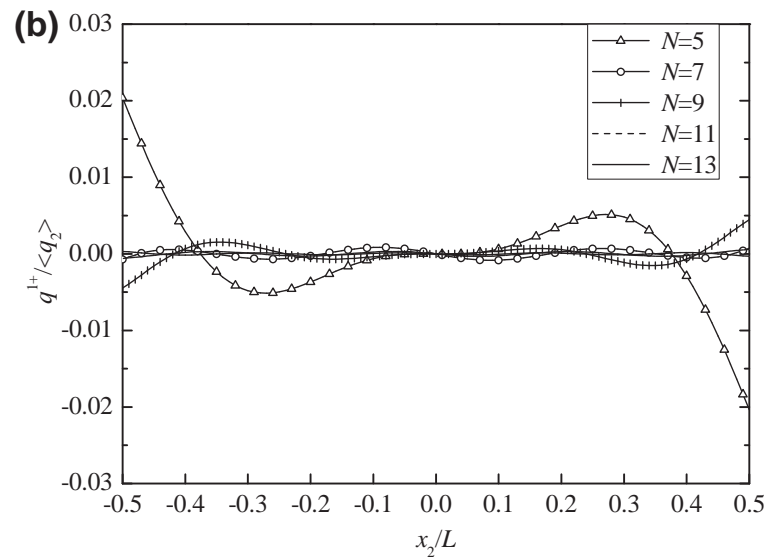

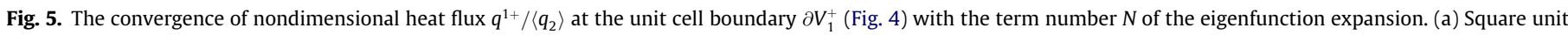
cell and (b) hexagonal unit cell.

Table 1

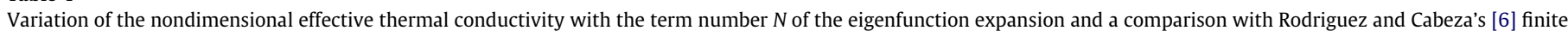
element results (square array, $k_{o} / k_{m}=1, R_{0} / R=0.2, \lambda=0.6$ ).

\begin{tabular}{|c|c|c|c|c|c|c|}
\hline \multirow[t]{3}{*}{$N$} & \multicolumn{3}{|c|}{$k_{e} / k_{m}$ (perfect interface, $\beta=0$ ) } & \multicolumn{3}{|c|}{$k_{e} / k_{m}$ (imperfect interface, $\beta=0.0572$ ) } \\
\hline & $k_{r} / k_{m}=2.4$ & $k_{r} / k_{m}=100$ & $k_{r} / k_{m}=\sqrt{240}$ & $k_{r} / k_{m}=2.4$ & $k_{r} / k_{m}=100$ & $k_{r} / k_{m}=\sqrt{240}$ \\
\hline & $k_{\theta} / k_{m}=100$ & $k_{\theta} / k_{m}=2.4$ & $k_{\theta} / k_{m}=\sqrt{240}$ & $k_{\theta} / k_{m}=100$ & $k_{\theta} / k_{m}=2.4$ & $k_{\theta} / k_{m}=\sqrt{240}$ \\
\hline 1 & 3.53845 & 2.37832 & 3.48229 & 2.56219 & 1.92313 & 2.53435 \\
\hline 3 & 3.39699 & 2.32789 & 3.34636 & 2.47113 & 1.88798 & 2.44625 \\
\hline 5 & 3.38882 & 2.32507 & 3.33853 & 2.46493 & 1.88565 & 2.44026 \\
\hline 7 & 3.38912 & 2.32515 & 3.33881 & 2.46489 & 1.88563 & 2.44021 \\
\hline 9 & 3.38915 & 2.32516 & 3.33884 & 2.46489 & 1.88563 & 2.44021 \\
\hline 11 & 3.38915 & 2.32516 & 3.33884 & 2.46489 & 1.88563 & 2.44021 \\
\hline FEM [6] & 3.37 & 2.38 & 3.32 & 2.46 & 1.92 & 2.44 \\
\hline
\end{tabular}


Table 2

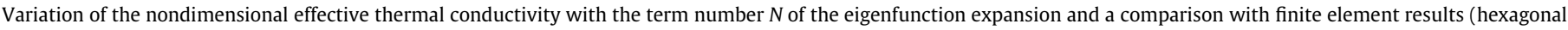
array, $k_{o} / k_{m}=1, R_{0} / R=0.2, \lambda=0.6$ ).

\begin{tabular}{|c|c|c|c|c|c|c|}
\hline \multirow[t]{3}{*}{$N$} & \multicolumn{3}{|c|}{$k_{e} / k_{m}$ (perfect interface, $\beta=0$ ) } & \multicolumn{3}{|c|}{$k_{e} / k_{m}($ imperfect interface, $\beta=0.0572)$} \\
\hline & $k_{r} / k_{m}=2.4$ & $k_{r} / k_{m}=100$ & $k_{r} / k_{m}=\sqrt{240}$ & $k_{r} / k_{m}=2.4$ & $k_{r} / k_{m}=100$ & $k_{r} / k_{m}=\sqrt{240}$ \\
\hline & $k_{\theta} / k_{m}=100$ & $k_{\theta} / k_{m}=2.4$ & $k_{\theta} / k_{m}=\sqrt{240}$ & $k_{\theta} / k_{m}=100$ & $k_{\theta} / k_{m}=2.4$ & $k_{\theta} / k_{m}=\sqrt{240}$ \\
\hline 1 & 3.28655 & 2.29749 & 3.24057 & 2.45997 & 1.88522 & 2.43551 \\
\hline 3 & 3.24219 & 2.28167 & 3.19782 & 2.43685 & 1.87608 & 2.41310 \\
\hline 5 & 3.24332 & 2.28203 & 3.19891 & 2.43693 & 1.87611 & 2.41318 \\
\hline 7 & 3.24337 & 2.28204 & 3.19896 & 2.43691 & 1.87611 & 2.41317 \\
\hline 9 & 3.24337 & 2.28204 & 3.19896 & 2.43691 & 1.87611 & 2.41317 \\
\hline 11 & 3.24337 & 2.28204 & 3.19896 & 2.43691 & 1.87611 & 2.41317 \\
\hline FEM & 3.24 & 2.29 & 3.20 & 2.44 & 1.88 & 2.41 \\
\hline
\end{tabular}

cient engineering closed-form formula. Further discussions refer to Fig. 6.

Tables 1 and 2 show that the present first-order approximation possesses a good engineering accuracy. To verify this observation, a lot of numerical computations are made. As an example, consider the case without an isotropic core in the fiber $\left(R_{0} / R=0\right)$ and take the nondimensional effective thermal conductivity of the fiber, $k_{f} / k_{m}=\sqrt{240}$, two values of the thermal contact conductivity, $h=10 k_{m} / R(\beta=0.05)$ and $h=\infty(\beta=0)$. A comparison of the present first-order approximation (EEVM, $N=1)$ with Hasselman's results [15] and the present high-order numerical solutions (EEVM, $N=11$ ) is depicted in Fig. 6a for a square array and in Fig. 6b for a hexagonal array, where $k_{e} / k_{m}$ is the nondimensional effective thermal conductivity of the composite, $\lambda$ is the fiber volume fraction, $N$ is the taken eigenfunction expansion term number, $E E V M=$ eigenfunction expansion-variational method. It is seen that the first-order approximate results have a good accuracy compared with the high-order numerical results, especially for a hexagonal array.

It should be pointed out that Hasselman's model and the present calculations (for the square and hexagonal arrays of fibers) are related to three different distributions of fibers. Hasselman et al. [15] wrote that because no interaction between fibers in their model was assumed, their effective thermal conductivity formula would be valid for dilute volume fractions only. We find that their model reflects interaction between fibers in a statistical sense and can be regarded as an idealized even distribution of fibers. In present calculations, the square and hexagonal arrays of fibers are considered. Fig. 6 shows that for a small (dilute) volume fraction of fibers, the three fiber distributions give almost identical results, which shows interaction between fibers is weak. However, for a large fiber volume fraction, the difference between the nondimensional effective thermal conductivity for the three fiber distributions becomes lager and larger with the increase of the fiber volume fraction. The phenomenon shows that interaction between fibers becomes strong and the effect of the fiber distribution becomes significant. In periodic fiber arrays, the hexagonal array appears the closest to the idealized even distribution and Fig. 6b shows the effective thermal conductivities for the two fiber distributions are very close. Besides, Eq. (36) indicates that a composite with high thermal conductivity fibers and imperfect interfaces can be approximately equivalent to the one with lower thermal conductivity fibers and perfect interfaces. Such a fact can explain the phenomenon observed from Fig. 6: the fiber interaction for $\beta=0.05$ is weaker than that for $\beta=0$, as a result, Hasselman's results and the high-order numerical results (EEVM, $N=11$ ) for $\beta=0.05$ are closer together than those for $\beta=0$.

Now discuss the effects of the cylindrical orthotropy of the fiber and the existence of the isotropic core.

The distributions of the nondimensional heat flux $q_{2} /\left\langle q_{2}\right\rangle$ along the $x_{2}$-axis (see Fig. 4 ) are depicted in Fig. 7 for a square array with different properties of the fiber and core. The parameters are taken as $\lambda=0.6, \beta=k_{m} /(2 h R)=0.05, N=11$. When no transversely isotropic core exists in the fiber, it is seen that the cylindrical orthotropy of the fiber has significant influence on the heat flux at the fiber center, but no influence on the heat flux in the matrix. The numerical results verify the conclusions obtained in the qualitative analysis that the radial and circumferential heat fluxes at $r=0$ are singular for $k_{r}>k_{\theta}$, finite for $k_{r}=k_{\theta}$ and zero for $k_{r}<k_{\theta}$. When a transversely isotropic core exists in the fiber, it is seen that the
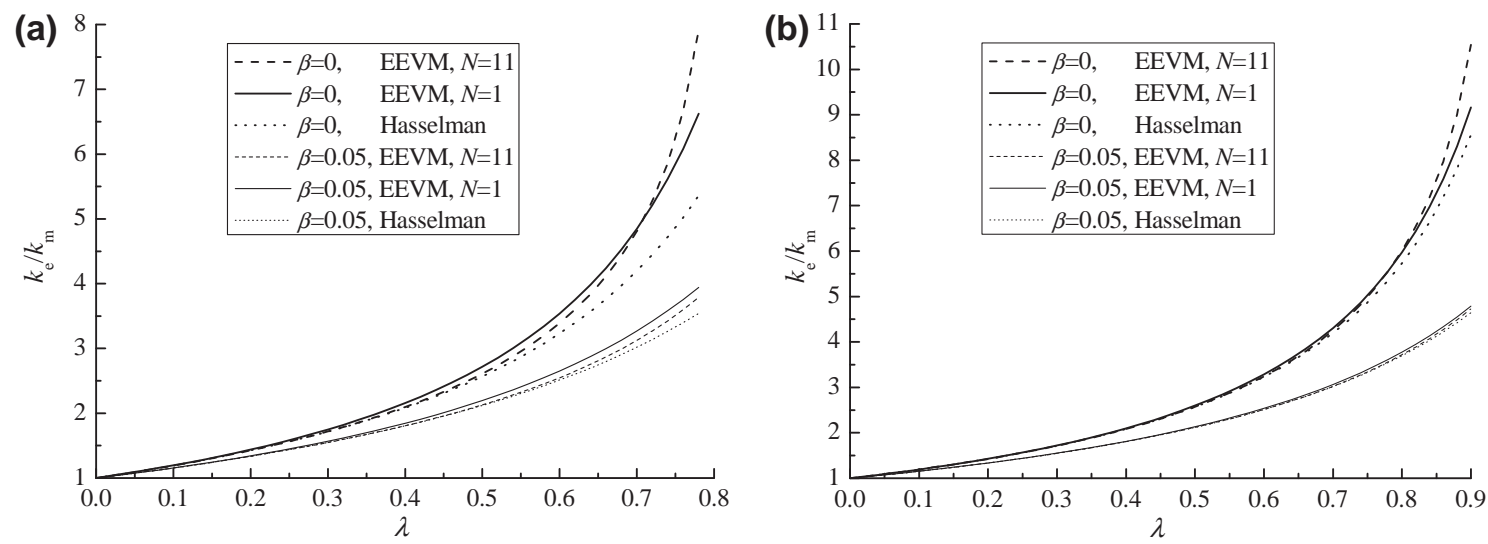

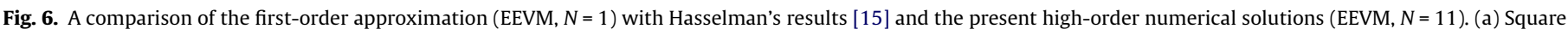
array and (b) hexagonal array. 

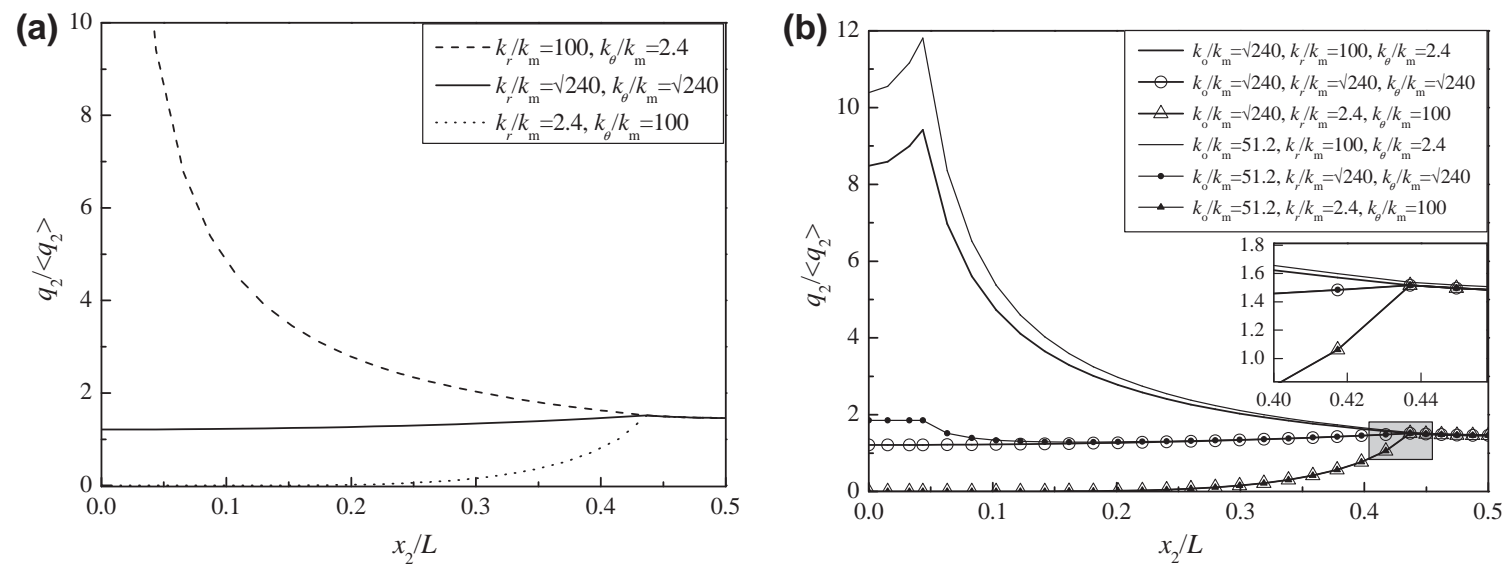

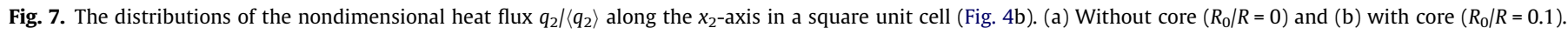

radial and circumferential heat fluxes at $r=0$ are finite, and an amplification effect of the radial orthotropy $\left(k_{r}>k_{\theta}\right)$ and a shielding effect of the circumferential orthotropy $\left(k_{r}<k_{\theta}\right)$ on the heat flux in the core are observed.

The transversely isotropic core is made up of randomly oriented graphite basal planes, a possible range of whose transverse conductivity can be estimated by the thermal conductivity of the single crystal graphite, that is $2.4<k_{\mathrm{o}} / k_{m}<100$. The effect of the nondimensional conductivity of the core on the effective conductivity of the composite is depicted in Fig. 8 for a square array, $\lambda=0.7$, and $R_{0} / R=0.2$. It is assumed that a perfect interface exists between the fiber and matrix, that is $h=\infty(\beta=0)$. It is seen that the influence of the conductivity of the core on the effective conductivity is significant when $k_{r}>k_{\theta}$. The value of the nondimensional conductivity of the core is $\sqrt{240}$ at the intersection point of the three curves. The reason is that the cylindrical orthotropy of the fiber have no influence on the effective conductivity only when $k_{o} / k_{m}=\sqrt{k_{\theta} k_{r}} / k_{m}=\sqrt{240}$.

The effect of the core radius on the effective conductivity is depicted in Fig. 9 for a square array. The parameters are taken as $\lambda=0.7, k_{\mathrm{o}} / k_{m}=(100+2.4) / 2=51.2, h=\infty(\beta=0)$. It is seen that the effect of the core radius on the effective conductivity depends on the cylindrical orthotropy of the fiber. If $k_{r} \gg k_{\theta}$, the influence of the core radius on the effective conductivity is significant when $R_{0} / R<0.1$, but becomes slight with the increase of $R_{0} / R$. If $k_{r} \ll k_{\theta}$,

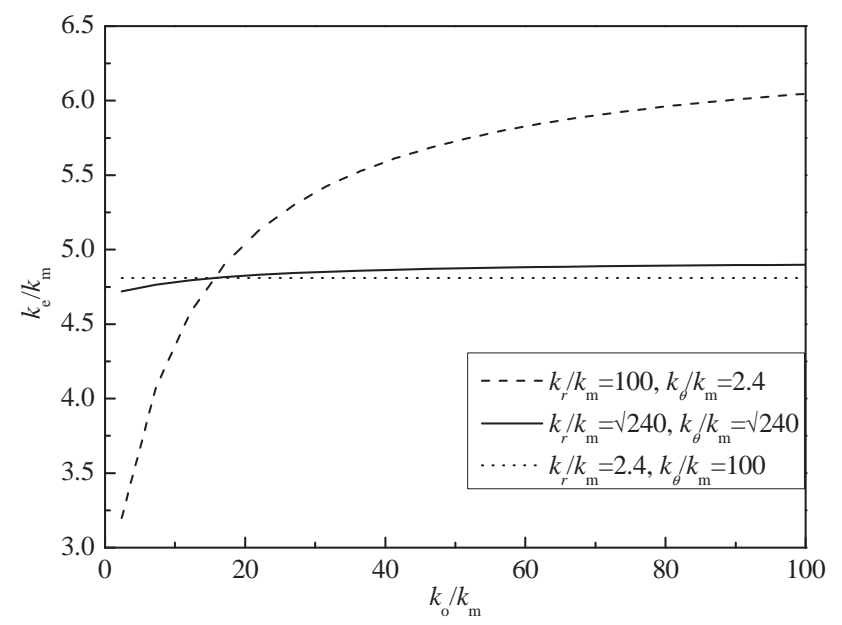

Fig. 8. The effect of the nondimensional conductivity $k_{o} / k_{m}$ of the core on the effective conductivity $k_{e} / k_{m}$ of the composite.

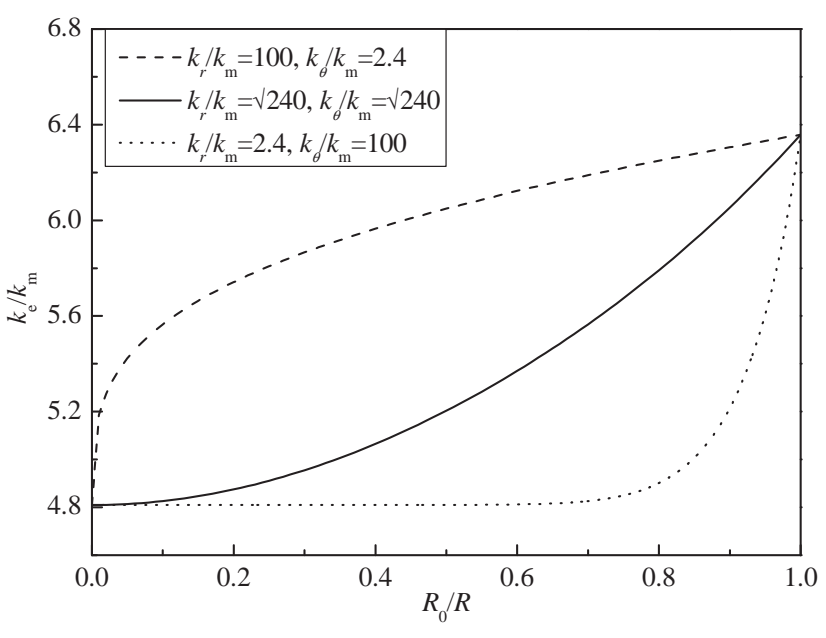

Fig. 9. The effect of the nondimensional radius $R_{0} / R$ of the core on the effective conductivity $k_{e} / k_{m}$ of the composite.

such influence is very trivial when $R_{0} / R<0.8$, but becomes significant when $R_{0} / R>0.8$.

\section{Concluding remarks}

By introducing an appropriate coordinate transformation, the governing differential equation for two-dimensional steady-state heat conduction in a cylindrically orthotropic solid is converted into a harmonic equation and a complex variable method is developed. The field variables for composites with doubly periodic arrays of cylindrically orthotropic fibers are formulated by complex potentials, which are expanded into eigenfunction series in a unit cell. Then by using a generalized variational functional which incorporates the periodic boundary condition of a unit cell, an eigenfunction expansion-variational method based on a unit cell is developed to solve such problems. A convergence analysis and a comparison with finite element calculations are made to demonstrate the validity and efficiency of the present method. On the other hand, the present method can provide reference results for other numerical and approximate methods.

The present method allows a profound understanding of the microstructure, microscopic fields, macroscopic effective 
properties and their interrelations for such composites. An engineering equivalent parameter $\eta$ is found and the parameter can reflect the overall influence of the thermal conductivities of the matrix and fibers as well as the interfacial characteristic on the effective thermal conductivity of the composite with a good engineering accuracy. The equivalent parameter greatly simplifies the complicated relation of the effective thermal conductivity to the internal structure of a composite.

By using the equivalent parameter $\eta$, the present solutions provide a unified first-order approximation formula of the effective thermal conductivity for different microstructural characteristics. A comparison with high-order solutions shows that the unified first-order approximation formula possesses good engineering accuracy and can serve as an engineering closed-form formula.

If no transversely isotropic core exists in the fiber, the heat flux at the center of the fiber is singular when the radial conductivity $k_{r}$ is larger than the tangential conductivity $k_{\theta}$, is finite when $k_{r}=k_{\theta}$ and is zero when $k_{r}<k_{\theta}$.

If a transversely isotropic core exists in the fiber, the cylindrical orthotropy of the fiber have no influence on the effective conductivity of the composites when the conductivity of the core is equal to the geometric mean of the radial and tangential conductivities of the fiber. If the radius of the core is relatively small, the influence of the radius and conductivity of the core is significant when $k_{r} \gg k_{\theta}$, and the influence of the core is shielded when $k_{r} \ll k_{\theta}$.

\section{Acknowledgements}

The work is supported by the National Natural Science Foundation of China under Grant NNSFC 90716002, and The Open Fund of LNM.

\section{References}

[1] D. Gay, S.V. Hoa, Composite Materials: Design and Applications, second ed., CRC Press, New York, 2007.

[2] R.M. Christensen, J. Mech. Phys. Solids 42 (1994) 681-695.

[3] Z. Hashin, Mech. Mater. 8 (1990) 293-308.

[4] W.B. Avery, C.T. Herakovich, J. Appl. Mech. 53 (1986) 751-756.

[5] T.W. Knott, C.T. Herakovich, J. Compos. Mater. 25 (1991) 732-759.

[6] A.C. Rodriguez, J.M.G. Cabeza, J. Compos. Mater. 33 (1999) 984-1001.

[7] J.Q. Tarn, J. Elasticity 69 (2002) 1-13.

[8] T.C.T. Ting, Int. J. Solids Struct. 37 (2000) 401-409.

[9] J.Q. Tarn, H.H. Chang, Int. J. Solids Struct. 45 (2008) 303-319.

[10] I. Tsukrov, B. Drach, Int. J. Solids Struct. 47 (2010) 25-33.

[11] T. Chen, G.J. Dvorak, Y. Benveniste, J. Appl. Mech. 59 (1992) 539-546.

[12] S. Yang, F.G. Yuan, Int. J. Solids Struct. 32 (1995) 3603-3628.

[13] L.H. You, Comput. Mater. Sci. 29 (2004) 49-66.

[14] Y. Benveniste, T. Chen, G.J. Dvorak, J. Appl. Phys. 67 (1990) 2878-2884.

[15] D.P.H. Hasselman, K.Y. Donaldson, J.R. Thomas Jr., J. Compos. Mater. 27 (1993) 637-644.

[16] T. Chen, H.Y. Kuo, J. Appl. Phys. 98 (2005) 033716.

[17] Z.H. Xia, Y.F. Zhang, F. Ellyin, Int. J. Solids Struct. 40 (2003) 1907-1921.

[18] M. Jiang, I. Jasiuk, M. Ostoja-Starzewski, Comput. Mater. Sci. 25 (2002) 329-338.

[19] P. Yan, C.P. Jiang, F. Song, X.H. Xu, Chinese J. Aeronaut. 23 (2010) 54-60

[20] P. Yan, C.P. Jiang, Int. J. Mod. Phys. B 23 (2009) 1689-1694. 